\title{
Non oso o non mi oso?
}

\author{
Massimo Cerruti
}

PUBBLICATO: 21 FEBBRAIO 2020

\section{Quesito:}

Alcuni lettori chiedono informazioni sulla correttezza e sulla caratterizzazione regionale dell'uso di osarsi come verbo pronominale, in costruzioni come non mi oso o non mi oso insistere.

\section{Non oso o non mi oso?}

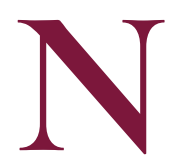
ellitaliano contemporaneo, l'uso di osarsi come verbo pronominale è caratteristico, seppur non esclusivo, di varietà nord-occidentali, e risulta particolarmente diffuso in Valle d'Aosta, Piemonte e Liguria. Specialmente in queste regioni, infatti, osarsi può comparire in luogo dell'equivalente panitaliano osare tanto nell'uso assoluto, come ad es. in non mi oso ("non oso"), quanto con un'infinitiva, come ad es. in non mi oso insistere ("non oso insistere"). L'uso di osarsi come verbo pronominale non è attestato nei dizionari dell'italiano, che annoverano soltanto il corrispondente non pronominale osare (si vedano, fra gli altri, GDLI, GRADIT, Sabatini-Coletti e Vocabolario Treccani).

Usi del tipo di non mi oso scoprire nel senso di "non oso scoprirmi", attestati in dizionari dell'italiano (ancorché ritenuti impropri; v. ad es. GDLI, s.v. osare), rappresentano una fattispecie diversa: in questi casi, invero, l'elemento pronominale è un argomento di scoprire (e ad essere considerata impropria è la sua risalita).

Nell'uso regionale, invece, il pronome è lessicalizzato con il verbo; tant'è che nel senso di "non oso scoprirmi" si può avere non mi oso scoprirmi, in cui il proclitico è parte stessa del verbo osarsi (non mi oso) e soltanto il pronome in enclisi sull'infinito è argomentale (scoprirmi).

Ancora di altro tipo, ovviamente, sono casi come ció che si osa immaginare o cose che non si osano dire, non marcati regionalmente, in cui il pronome si ha valore impersonale.

L'uso di osarsi in varietà nord-occidentali di italiano è appoggiato ai dialetti dell'area, in buona parte dei quali il significato di "osare" è reso infatti con un verbo pronominale. Consultando ad esempio la carta I64I dell'Atlante linguistico ed etnografico dell'Italia e della Svizzera meridionale (Sprach- und Sachatlas Italiens und der Südschweiz), è possibile osservare come vari dialetti del Nord-Ovest presentino per "osare" forme del tipo di incaláse/incalése o ancaláse/ancalése (di etimo discusso; v. ad es. Manlio Cortelazzo, Carla Marcato, I dialetti italiani. Dizionario etimologico, UTET, Torino 2004, s.v. incaláse; Anna Cornagliotti, a cura di, Repertorio etimologico piemontese, Centro studi piemontesi, Torino 20I5, s.v. ancalé/in-), che incorporano evidentemente l'elemento pronominale se ("si"). Forme di questo stesso tipo sono attestate anche nei dizionari dei dialetti dell'area, e talvolta figurano a lemma insieme alle varianti prive di pronome; per l'ambito piemontese, si vedano ad esempio incallêe, incallesi nel Dizionario del dialetto valsesiano di Federico Tonetti (Varallo i894, rist. Bologna 1967) e ancalé, ancalesse nel Vocabolario piemontese-italiano e italiano-piemontese di Michele Ponza (Pinerolo I877, rist. Torino I967).

La coesistenza di forme con e senza elemento pronominale caratterizza altresì le rispettive varietà regionali di italiano, in cui l'alternanza fra osarsi e osare correla per lo più con fattori sociali e situazionali: generalmente, la prima forma compare più di frequente in varietà popolari e di registro informale, che sono oltretutto le più sensibili all'influenza del sostrato dialettale, mentre la seconda 
rappresenta la variante di uso medio e ha perciò una distribuzione meno marcata. Si vedano qui alcuni esempi, provenienti o dal parlato colloquiale o dal parlato grafico della comunicazione elettronica: $s i$ oserebbe anche ma non ci sta col tempo (parlato spontaneo, Torino; Massimo Cerruti, Strutture dellitaliano regionale, Frankfurt am Main, Lang, 2009, p. I2I); ora ke lui è tornato a Bologna e io a Imperia continuo a pensare a lui, ma nn mi oso fargli gli squilli (Corpus ItWac), la classica domanda (di chi si osa) è "chi fa l'uomo e chi la donna?" (Forum di discussione https://wrww.skyscrapercity.com). Le occorrenze del fenomeno in produzioni scritte di altro tipo sono soltanto sporadiche e il più delle volte riscontrabili in testi a circolazione limitata, come, fra gli altri, i periodici a diffusione locale; es. questo genere di centro d'ascolto non riesce a intervenire in situazioni in cui le persone non si osano presentarsi ("Gazzetta d'Asti", Supplemento, 8/7/2016, p. 3), sapeva della mia assenza e poi non si osava più chiedere ("Saluzzo Oggi”, I9/ı/20Iо, p. 4 https://www.giornalidelpiemonte.it).

In conclusione, l'uso di osarsi invece di osare è caratteristico di alcune varietà geografiche d'italiano, e nemmeno in ambito regionale, essendo raro in testi scritti e formali, può ritenersi un tratto di norma.

\section{Cita come:}

Massimo Cerruti, Non oso o non mi oso?, "Italiano digitale", 2019, XII, 2020/1 (gennaio-marzo)

DOI: $10.35948 / 2532-9006 / 2020.3234$

Copyright 2019 Accademia della Crusca

Pubblicato con licenza creative commons CC BY-NC-ND 